\title{
Transporte y su integración con el entorno urbano: ¿cómo incorporamos los beneficios de elementos urbanos en la evaluación de proyectos de transporte?
}

\author{
Isidora Navarro. Pontificia Universidad Católica de Chile, Santiago, Chile. \\ Patricia Galilea. Pontificia Universidad Católica de Chile, Santiago, Chile. \\ Rocío Hidalgo. Pontificia Universidad Católica de Chile, Santiago, Chile. \\ Ricardo Hurtubia. Pontificia Universidad Católica de Chile, Santiago, Chile.
}

RESUMEN | Los proyectos de transporte urbano tienen un gran potencial para influir en la configuración y carácter del espacio público de nuestras ciudades. Actualmente, la evaluación social de proyectos de transporte en Chile considera atributos principalmente relacionados al transporte. Sin embargo, la implementación de proyectos de transporte puede llegar a entregar beneficios adicionales al entorno, los cuales no están siendo considerados por las metodologías actuales, aunque sus costos de implementación y de mantención sí son considerados. Este artículo propone una metodología basada en el uso de preferencias declaradas (PD) para evaluar en conjunto variables urbanas de espacio público y de transporte, considerando los siguientes tres atributos: existencia de áreas verdes, ciclovía y corredor de buses. Estos atributos resultaron tener una valoración positiva en todos los sectores, reafirmando la necesidad de considerar los impactos de los proyectos de transporte en la configuración y calidad de los espacios públicos urbanos. Por lo tanto, se propone incluir estos beneficios positivos en futuras evaluaciones de proyectos.

PALABRaS ClAVE | espacio público, infraestructura urbana, movilidad.

ABSTRACT | Urban transport projects have great potential to influence public space and the way we understand the city. Nowadays, the design and cost-benefit analysis of transport infrastructure projects in Chile consider attributes mainly related to transport. However, the implementation of transport projects can also give additional benefits to the environment, which are not being considered by current methodologies, although their costs of implementation and maintenance are included. This article proposes a methodology using stated preferences $(S P)$ to evaluate urban attributes in the transport planning context, considering three urban attributes: Existence of green areas, bike paths and bus corridors. The attributes were found to have a positive valuation in all sectors, reaffirming the importance of the qualities and characteristics of urban spaces. Therefore, these positive benefits should be incorporated in future evaluations.

KEYWORDs | public space, urban infrastructure, mobility. 


\section{Introducción}

Toda ciudad promueve y acoge múltiples desplazamientos de sus habitantes, en patrones de movilidad, que se ven complejizados debido al acelerado crecimiento urbano. Por otro lado, son muchas las ciudades que cuentan con presupuestos acotados, más aún si pertenecen a países en vías de desarrollo. En Chile existe una larga experiencia de uso de la evaluación social de proyectos de infraestructura de transporte para tomar decisiones de inversión. Sin embargo, esta metodología presenta limitaciones, ya que considera, esencialmente, beneficios y costos directos del transporte, como son reducciones del tiempo de viaje, algunas externalidades, costos de construcción y operacionales. En cuanto a aquellos proyectos de infraestructura en transporte que incluyen propuestas integradas de transformación de su entorno urbano, ellos se encuentran en desventaja frente a otros que no las incorporan, porque la metodología de evaluación social no considera eventuales beneficios adicionales de mejora del espacio público involucrado (y, en consecuencia, a sus usuarios y habitantes), aunque sí toma en cuenta los costos de construcción y mantención de esas propuestas. Esta investigación entrega luces sobre la importancia de considerar algunos elementos urbanos y sus beneficios para formular mecanismos de evaluación integrada de proyectos de transporte y espacio público.

Existen elementos de la ciudad que influyen y traen consecuencias, beneficios o costos indirectos, en más de un ámbito y en diferentes escalas. Ejemplos clásicos de esto son la infraestructura para bicicletas, las áreas verdes y facilidades peatonales, elementos que suelen formar parte del espacio público urbano. En los últimos años, la bicicleta se ha convertido en una real opción como modo de transporte en Chile. De hecho, según la Encuesta Origen Destino 2012 (Secretaría de Planificación de Transporte [SECTRA], 2014), la bicicleta experimentó una tasa de crecimiento entre los ańos 2001 y 2012 de un 6,8\% anual, duplicándose la cantidad de viajes en el periodo. El incremento en el uso de este modo ayuda a disminuir la congestión, contaminación, y al mismo tiempo, trae beneficios a las personas (Gotschi, 2011; Krizec, 2007). Por otro lado, las áreas verdes también se están haciendo cada día más importantes, pues no solo influyen en la configuración y habitabilidad de los espacios públicos, sino también en el incremento de la calidad de vida de los habitantes de una ciudad (Krekel, Kolbe \& Wüstemann, 2016; Mullaney, Lucke \& Trueman, 2015).

El problema radica en que no se conoce el beneficio o valoración por (mejoras en) los atributos del espacio público, por lo que en Chile no hay incentivos claros y medibles para invertir en mejoramientos del entorno de los proyectos de transporte. Mackie, Worsley y Eliasson (2014) hacen una revisión y comparación de las características y atributos considerados en las evaluaciones de proyectos en siete países. Estos países usan metodologías que incorporan atributos no monetizados en las evaluaciones sociales de los proyectos (cost-benefit analysis): los "impactos económicos más amplios" (wider economic impacts) como, por ejemplo, aquellos relacionados con las economías de aglomeración, el paisaje, mejoras de espacios públicos, preservación de patrimonio y consecuencias en la salud física o psicológica de los usuarios. 
Hasta ahora se han aplicado metodologías con un enfoque principalmente cualitativo para incluir los elementos urbanos y metodologías con un enfoque cuantitativo para las evaluaciones de proyectos, desde la valoración del ahorro en tiempos de viaje (Mackie, Jara-Díaz, \& Fowkes, 2001) hasta distintas formas de pensar la ciudad y sus espacios públicos (Gehl \& Svarre, 2013; Miralles-Guasch, 2002; Rueda, 2013).

En los últimos años se le ha dado mucha importancia al bienestar de las personas, pero no se han desarrollado suficientes metodologías integradas de diseño urbano y de transporte, condición hoy fundamental para un incremento sustentable de la calidad de vida de las personas o well-being (e.g. Delbosc, 2012; Rueda, 2013; Vella-Brodrick \& Stanley, 2013). Dado el contexto de crecimiento de la población urbana, la necesidad de hacer cada vez más sustentables y amigables las ciudades, y el interés por parte de distintas autoridades chilenas de profundizar en estudios al respecto, surge la necesidad de nuevas investigaciones y metodologías que ayuden a estudiar la ciudad de forma integral, explorando especialmente las relaciones entre transporte, espacio público y desarrollo urbano.

Esta investigación plantea que es posible aplicar una única metodología para evaluar los diversos atributos que caracterizan a los espacios públicos urbanos e identificar la disposición al pago por estos atributos. Esto se puede llevar a cabo a través de un experimento de preferencias declaradas (PD), una metodología ampliamente utilizada para conocer las preferencias de las personas por distintos atributos presentes en un conjunto de alternativas usando un modelo de elección discreta (Louviere, Hensher \& Swait, 2000; Ortúzar \& Willumsen, 2011). El objetivo de esta investigación es valorar cuantitativamente determinados atributos urbanos que pudieran mejorar el espacio público de la calle, en particular en el caso de ejes que pueden acoger corredores de transporte público y ciclovías. Gracias a la valoración de estos atributos, se propone el desarrollo de una metodología de evaluación basada en encuestas de PD con apoyo de imágenes para la formulación de alternativas, instrumento que se pone a prueba, a modo de caso de estudio, en simulaciones de transformación del uso y configuración del espacio público de cuatro avenidas de cuatro entornos diferentes en Santiago de Chile.

Algunas investigaciones de PD con el uso de imágenes se han llevado a cabo en distintos ámbitos. Se han valorado algunos atributos de vivienda y barrio (Torres, Greene \& Ortúzar, 2013), barrios patrimoniales (Bonet, 2014) y la percepción de seguridad en barrios de escasos recursos (Sillano \& Ortúzar, 2005). Desde una mirada de espacios públicos, se valoró la modificación de una autopista (Grisolía, López \& Ortúzar, 2014), la regeneración de proyectos en áreas urbanas (Strazzera, Cherchi \& Ferrini, 2010) y preferencias urbanas en el espacio peatonal (Hurtubia, Guevara \& Donoso, 2015).

En el caso de esta investigación, para la formulación de alternativas se consideraron variaciones en tres atributos principales de la calle: áreas verdes, ciclovía y corredor de buses. Se crearon distintas imágenes para cada una de las avenidas estudiadas, que tenían diferentes combinaciones de estos atributos, junto con un valor de arriendo ficticio. A los encuestados se les presentaban seis preguntas con dos imágenes cada una, y tenían que elegir en cuál de ellas preferirían vivir, considerando que únicamente se produce una variación en el entorno y no en las 
características de su vivienda, con la excepción del valor de arriendo o venta. A partir de estas respuestas, es posible estimar modelos de elección discreta y conocer las preferencias de los usuarios por atributos urbanos en los distintos barrios, y al mismo tiempo cuánto estarían dispuestos a pagar por tenerlos en su entorno.

Este artículo está dividido en cuatro secciones. En la primera se explicará el marco teórico de la metodología ocupada. En la sección 2 se describirá la metodología y el proceso llevado a cabo para el desarrollo de la encuesta, para mostrar los resultados en la sección 3. Finalmente, se muestran las principales conclusiones y discusiones de esta investigación.

\section{Marco teórico}

La necesidad de integrar las diferentes disciplinas que intervienen en la planificación, diseño y construcción del espacio urbano es una realidad ampliamente explorada y reconocida en el ámbito teórico y académico, aunque no igualmente desarrollada a nivel de la práctica, en la evaluación e implementación de los proyectos de transformación del espacio urbano. Tal es el caso de los proyectos de transporte, que desde la incorporación del ferrocarril y los tranvías en la segunda mitad del siglo XIX, pasando por la expansión del automóvil en el segundo y tercer cuarto del siglo $\mathrm{xx}$, y asistiendo desde los años setenta del siglo pasado al resurgimiento de los sistemas de transporte colectivo, han constituido, a través de la construcción de sus infraestructura y la instalación de sus sistemas, elementos fundamentales de la estructura, carácter y habitabilidad de nuestras ciudades (Herce, 2009; Herce \& Magrinya, 2013; Miralles-Guasch, 2002).

El transporte es fundamental para las ciudades, en cuanto constituye el medio en que las personas se desplazan para satisfacer sus necesidades y llegar a sus destinos. Según Miralles-Guasch (2002), el transporte es un elemento necesario para el desarrollo de la ciudad moderna, sin el cual las ciudades no serían lo que son. No es posible pensar en ciudad separada del transporte. Son parte de una relación compleja entre personas, necesidades y servicios.

El desarrollo del transporte ha sido a la vez causa y consecuencia de los hábitos de desplazamiento de nuestros ciudadanos a lo largo de los últimos 150 años, integrando el carácter de nuestras ciudades e incidiendo, junto a otros factores, en la configuración y condiciones de habitabilidad del espacio público (Rueda, 2013). La ciudad es el lugar donde todas las relaciones sociales ocurren; es un lugar de encuentro con el otro, relaciones que el transporte restringe o promueve, según su grado de integración en la planificación y diseño de la ciudad como totalidad (Borthagaray, 2009). Es así que, dada su complejidad, la ciudad ha sido comúnmente abordada desde diferentes perspectivas disciplinares. Algunas se preocupan de la eficiencia urbana desde una mirada más cuantitativa; otras, de la forma y distribución de los atributos de una manera más cualitativa, pero no hay muchas instancias para poder planificar la ciudad en conjunto. Es necesario insistir en la planificación global de la ciudad, integrando particularmente las disciplinas que intervienen en la configuración del espacio público de la calle, espacio de encuentro, intercambio y desplazamientos (Ascher, 2005; Carmona, Tiesdell, Heath \& Oc, 2003; Herce, 2009). 
A partir de lo anterior, esta investigación se propone aplicar una metodología ampliamente usada hasta ahora en el ámbito del transporte, esta vez en conjunto con atributos que describen el espacio público de la calle. Para encontrar la valoración y modelar las preferencias de las personas por distintos atributos, la mayoría de los estudios son realizados a través de modelos de elección discreta. Son una forma de explicar y entender cómo las personas eligen una opción dentro de un conjunto determinado de alternativas; en este caso, imágenes de sus barrios con distintos atributos en su entorno. Cada alternativa que se les presenta puede ser descrita a partir de sus características e importancia relativa, en lo que se llama función de utilidad. La función de utilidad representa qué tan atractiva es esa alternativa para cada persona o qué tanto beneficio o "felicidad" le aporta. La cantidad de atributos considerados depende del modelador, por lo que siempre existe un componente de error asociado a atributos no considerados o información incompleta.

De manera sencilla, la función de utilidad $U_{i n}$ que una alternativa $i$ genera para una persona $n$ se puede describir de la siguiente manera:

$$
U_{\text {in }}=\beta_{1}^{n} * \text { atributo }_{1}+\cdots+\beta_{K}^{n} * \text { Atributo }_{k}+\text { error }
$$

La utilidad está compuesta por una serie de atributos, y cada uno de ellos tiene una importancia relativa para cada persona, descrita por el parámetro $ß$, que indica si le impacta de manera positiva o negativa en su felicidad/utilidad. Si el parámetro es positivo, indica que le reporta utilidad o "felicidad"; en cambio, si es negativo, significa que "disminuye su felicidad" el hecho de que esté presente.

En un inicio, el valor del parámetro ß para cada atributo es desconocido y es lo que se busca estimar con modelos de elección discreta. Los modelos de elección discreta se sustentan en la teoría de utilidad aleatoria (Domencich \& McFadden, 1975), en que los individuos siempre eligen la opción que les reporta mayor utilidad o "felicidad". Con base en esto, y con los datos recolectados a través de las encuestas, se hacen estimaciones para encontrar el conjunto de valores para los parámetros $\beta$ que reproduce de mejor forma la probabilidad de que una alternativa sea escogida por sobre la otra. En otras palabras, se determina el conjunto de parámetros que permite que el modelo se ajuste de la mejor forma posible a la realidad observada. ${ }^{1}$

Los distintos tipos de modelos de elección discreta se diferencian en la forma de considerar el error de la función de utilidad. El modelo más sencillo es el Modelo Logit Multinomial (MNL, por sus iniciales en inglés), que fue usado en esta investigación.

Por el lado de la recolección de datos, existen principalmente dos tipos de encuestas: basadas sobre elecciones reales hechas por los encuestados (Preferencias Reveladas, PR); y basadas en elecciones en situaciones hipotéticas (Preferencias Declaradas, PD) (véase Ortúzar, Martínez \& Varela, 2000). En términos generales, las encuestas de preferencias declaradas tratan de explicar el comportamiento de los usuarios a partir de cómo actuarían si se enfrentaran a situaciones hipotéticas, pero basadas en la vida real.

1 Para más detalles sobre el proceso de estimación, se sugiere revisar el trabajo de Ben-Akiva y Lerman (1985). 


\section{Metodología}

En esta sección se mostrará la generación y diseño de la encuesta realizada. Para mayor detalle, revisar Navarro (2016), donde se explican las experiencias anteriores en materia de metodologías y atributos considerados. El objetivo principal de hacer este experimento es la obtención de las preferencias de los usuarios por los diferentes atributos urbanos presentes en sus barrios.

\section{Características}

La encuesta es un experimento de PD en un contexto de elección residencial. Se realizó en línea y con el apoyo de imágenes reales de los sectores en que se llevaron a cabo las encuestas, ya que es la manera más fácil de mostrar espacios públicos y que sean entendidos de la misma manera por los encuestados. A los encuestados se les decía: "suponga que usted no es dueño de su vivienda y está considerando arrendar una idéntica a la que usted usa, pero en distintos contextos urbanos”. A continuación les aparecían seis preguntas y tenían que elegir entre dos imágenes que representaban distintas mejoras al entorno de sus barrios actuales.

\section{Selección de atributos relevantes}

La selección de los atributos fue un proceso importante en esta investigación. Esto, debido a que originalmente los modelos de ingeniería tienden a considerar cada atributo por separado, y la visión del urbanismo es completamente distinta. A través de una revisión de literatura relevante en el área de urbanismo, se logró entender la real importancia de diferentes aspectos urbanos y se identificaron los atributos o características involucradas en la creación de espacios y calles (Gehl \& Svarre, 2013; Jacobs, 1961; Miralles-Guasch, 2002). Paralelamente, se tuvo conversaciones con diferentes autoridades de transporte, las que manifestaron su interés por un atributo en particular: áreas verdes. Además, con el fin de relacionar los espacios públicos con transporte, se decidió incorporar una ciclovía y un corredor de buses.

Un aporte significativo al entorno de una calle y su paisaje, son los árboles. Una revisión de los beneficios y desafíos de los árboles en las calles (Mullaney, Lucke \& Treman, 2015), permite concluir que los árboles son un elemento integral de la vida urbana. Entregan una amplia gama de beneficios ambientales, sociales y económicos; e.g. aumentan la habitabilidad de ciudades, mejoran la calidad del aire, mitigan el ruido, promueven el contacto entre los residentes de una comunidad y animan la actividad física.

Por el lado de la movilidad, en los últimos años la bicicleta se ha convertido en una real opción como modo de transporte, y la ciclovía es una opción de infraestructura para promover y facilitar su uso. Según la Encuesta Origen Destino 2012 (SECTRA, 2014) para Santiago, la bicicleta duplicó su participación entre los modos de transporte entre los años 2001 y 2012. Existe además evidencia de que el desarrollo de infraestructura ciclista induce a un mayor uso de este modo de transporte (Dill \& Carr, 2003: Nelson \& Allen, 1997).

Desde otro punto de vista, se ha visto la necesidad e importancia de darle prioridad al transporte público a través de corredores de buses. De hecho, según la 
Encuesta Origen Destino 2012 (sectra, 2014), el 29,1\% de los viajes diarios en la Región Metropolitana de Santiago se realiza en transporte público, cifra levemente superior al porcentaje en transporte privado (28\%). Dada la cantidad de vehículos circulando en horas punta, y el impacto que tiene en el entorno la construcción de un corredor, surge como opción crear nuevos diseños o formas de implementar esta infraestructura para que complemente el espacio urbano, sin que rompa con las características actuales del sector.

\section{Selección de lugares}

Para la verificación de la metodología propuesta, se seleccionaron segmentos de cuatro avenidas de Santiago de Chile que fuesen ejes estructurantes del sistema de transporte público, con y sin corredores de buses construidos, y emplazadas en sectores de características socioeconómicas diversas. De esta manera, se seleccionaron segmentos de cuatro avenidas: Vitacura, Santa Rosa, Gran Avenida y Pajaritos (tabla 1).

TABLA I | Características de las comunas a las cuales pertenecen los sectores

\begin{tabular}{|l|l|c|c|c|c|}
\hline \multicolumn{1}{|c|}{ SECTOR } & COMUNA & $\begin{array}{c}\text { INGRESO AUTÓNOMO } \\
\text { PROMEDIO (*) } \\
\text { (CASEN 20I3, EN CLP) }\end{array}$ & $\begin{array}{c}\text { VIVIENDAS } \\
\text { (SII 2OIO, } \\
\text { EN CLP) }\end{array}$ & $\begin{array}{c}\text { POBLACIÓN } \\
\text { (EOD 2OI2) }\end{array}$ & $\begin{array}{c}\text { VEHÍCULOS } \\
\text { POR HOGAR } \\
\text { (EOD 2OI2) }\end{array}$ \\
\hline Vitacura & Vitacura & 2.645 .755 & 29.044 & 96.188 & 1,7 \\
\hline \multirow{2}{*}{ Santa Rosa } & La Granja & 475.140 & 28.338 & 130.272 & 0,37 \\
\cline { 2 - 6 } & San Ramón & 542.401 & 19.340 & 91.410 & 0,37 \\
\hline Gran Avenida & La Cisterna & 911.511 & 22.279 & 87.778 & 0,47 \\
\hline Pajaritos & Maipú & 848.133 & 144.390 & 515.375 & 0,5 \\
\hline
\end{tabular}

(*) INGRESO AUTÓNOMO: INGRESO POR CONCEPTO DE SUELdOS Y SALARIOS, EN PESOS CHILENOS (CLP).

FUENTE ELABORACIÓN PROPIA BASADA EN LA ENCUESTA DE CARACTERIZACIÓN SOCIOECONÓMICA (CASEN) (MiNisterio DE DESARRollo SOCIAL, 2OI3); SERViCiO DE IMPUESTOS INTERNOS SII, 2OIO); Y ENCUESTA ORIGEN DESTINO (EOD) 2012 (SECTRA, 2OI4)

Los cuatro sectores tienen características particulares y se diferencian en el nivel de ingreso de sus habitantes, lo que aporta a tener distintos puntos de vista y, al mismo tiempo, considerar personas de distintos sectores y características sociales.

\section{Encuesta piloto}

La encuesta piloto es parte de un proceso iterativo para la creación de un diseño experimental, el que requiere ir repitiendo pasos para obtener mejores resultados finales.

\section{Atributos y niveles}

Los atributos considerados fueron las áreas verdes, espacio peatonal, ciclovía, corredor de buses, valor de arriendo y tiempo de viaje. El diseño del experimento se generó utilizando el software Ngene (ChoiceMetrics, 2012), el cual permite crear diseños eficientes si se tienen los valores iniciales de los parámetros que se requiere estimar. 


\section{Resultados y aprendizajes}

En primer lugar, se observó que el espacio peatonal y tiempo de viaje no eran atributos estadísticamente significativos y fueron eliminados para el diseño de la encuesta final. La vereda es un espacio público complejo; se podría hacer un estudio completo dedicado a las distintas mejoras que se pueden realizar, y no estaba siendo bien representado en las imágenes. El tiempo de viaje es un atributo que depende de características que no están presentes en las alternativas -por ejemplo, el lugar de trabajo del encuestado- y era complejo entender la relación con las imágenes y precio de arriendo.

Algunas de las lecciones que se aprendieron con el trabajo realizado en la encuesta piloto son:

- No es conveniente incluir atributos que puedan ser contraintuitivos o difíciles de asociar a la pregunta que se está haciendo, como es el caso del tiempo de viaje.

- Es preferible que las imágenes tengan la misma composición si se quiere comparar escenarios.

- Es preferible que los atributos que se quiere variar estén visibles y no desplazados al trasfondo de la imagen.

- Es preferible disminuir la cantidad de atributos y el ruido en la imagen para su mejor comprensión.

- Es preferible que el encuestado pueda reconocer el lugar y se sienta identificado, de modo que la decisión sea real.

\section{Encuesta final}

Algunas de las características y modificaciones de la encuesta se hicieron sobre la base de los aprendizajes del proceso iterativo que se llevó a cabo con la encuesta piloto y otras pruebas.

\section{Atributos y niveles}

En este caso se consideraron los árboles, ciclovía, corredor de buses, arbustos segregadores y precio de arriendo como atributos. Se incorporaron cinco niveles al atributo 'precio' para lograr mayor sensibilidad al respecto. El encuestado, tras contestar la pregunta “¿cuánto paga de arriendo mensual?” o “¿en cuánto cree que podría arrendar su propiedad?”, va a observar variaciones de $\pm 10 \%$ y $\pm 15 \%$ respecto de su situación actual.

\section{Creación de imágenes y encuesta}

Se trató de mantener la composición de las imágenes igual para cada sector; i.e. que la estructura de la imagen fuera la misma, tomada desde el mismo punto y con puntos de fuga similares. Las variaciones o incorporaciones en la imagen se realizaron solo en el costado derecho de esta, con el fin de que los encuestados fueran capaces de reconocer su sector manteniendo constante el costado izquierdo. En la figura 1 se pueden ver las imágenes originales y modificadas de los cuatro sectores. El orden es Vitacura, Santa Rosa, Gran Avenida y, finalmente, Pajaritos. 
FIGURA I Imagen original y ejemplo de imagen modificada de cada uno de los sectores
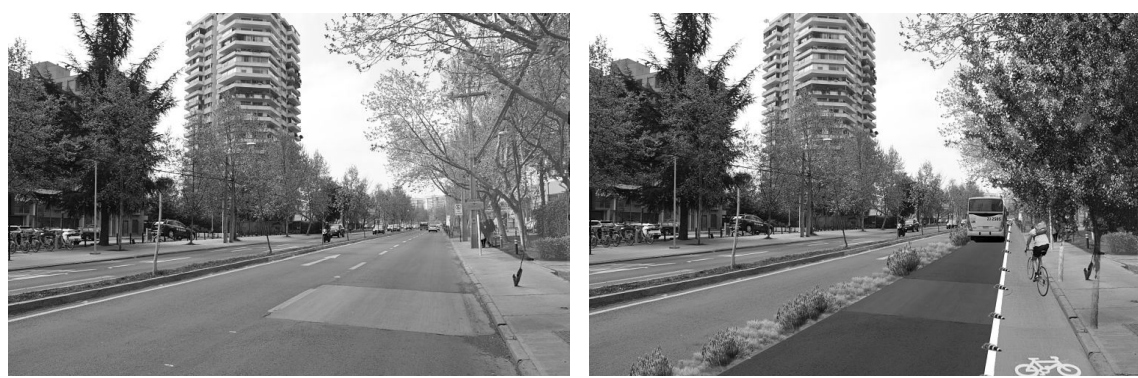

Vitacura

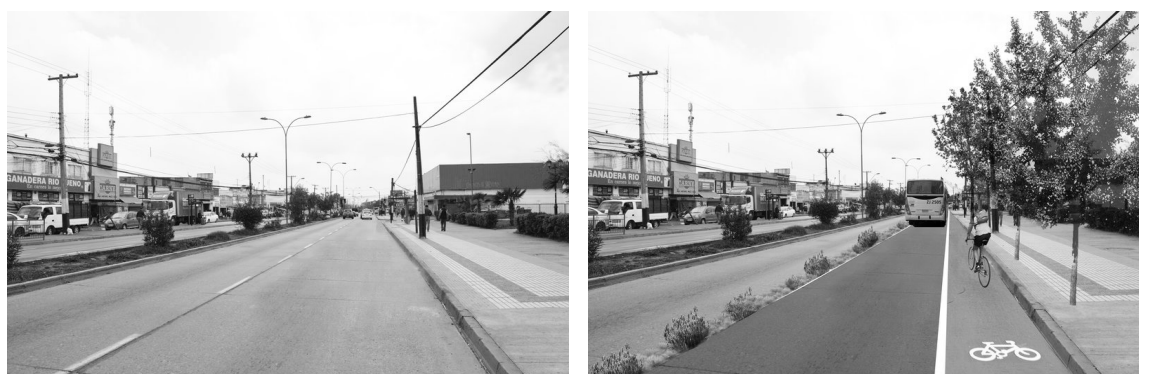

Santa Rosa
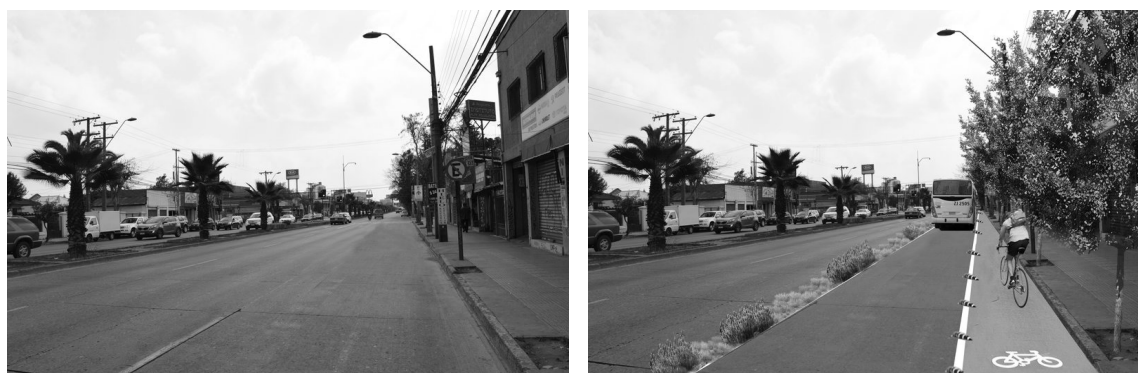

Gran Avenida
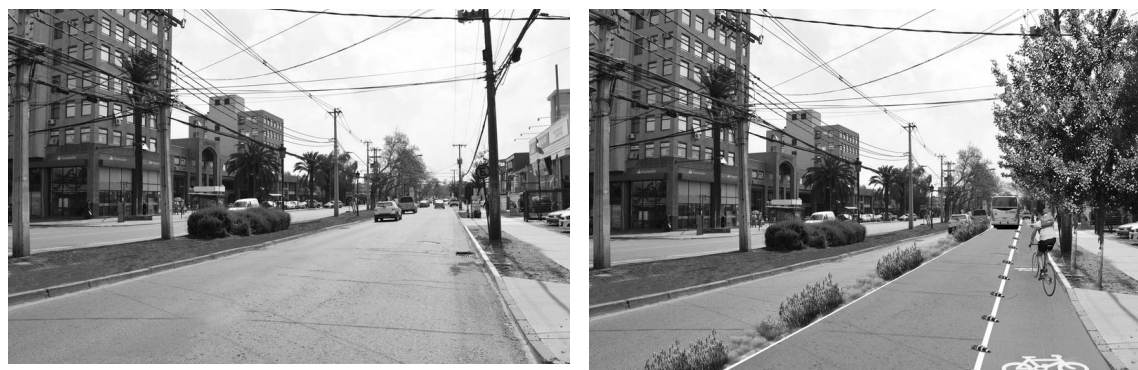

Pajaritos

FUENTE ELABORACIÓN PROPIA 
Las situaciones de elección de la encuesta final se ven con el mismo formato para los cuatro lugares. En las figuras 2 y 3 se presenta un ejemplo de dos de ellas.

FIGURA 2 | Ejemplo de situación de elección del sector Vitacura

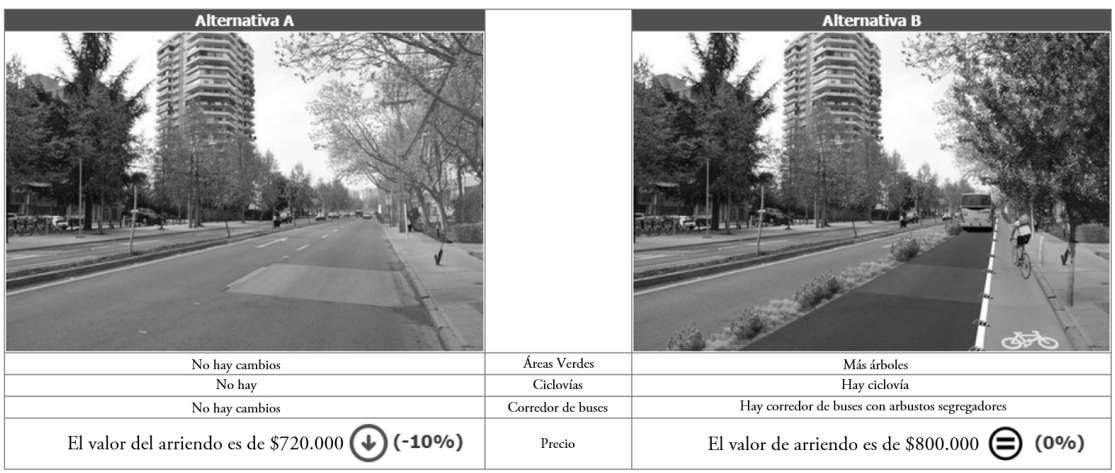

FUENTE ELABORACIÓN PROPIA

FIGURA 3 Ejemplo de situación de elección del sector Pajaritos

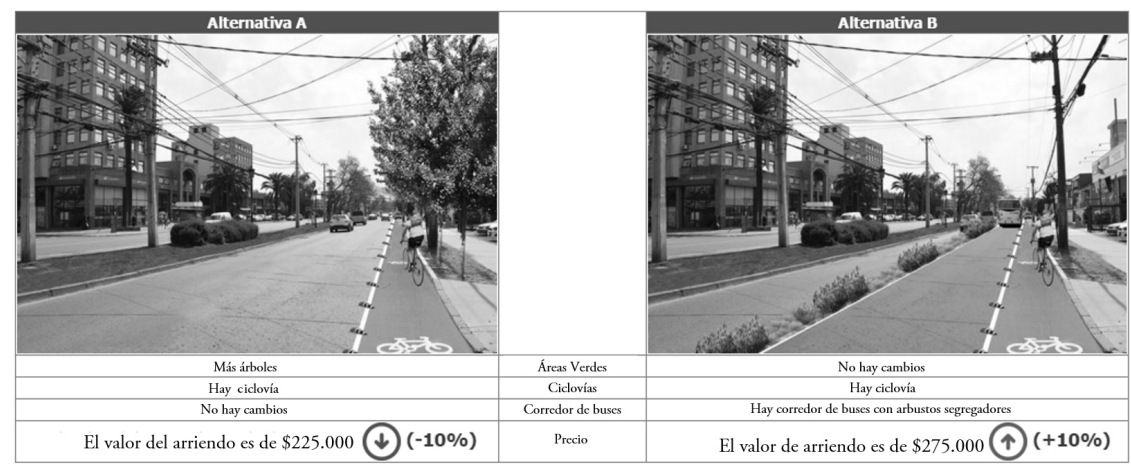

FUENTE ELABORACIÓN PROPIA

\section{Resultados}

A continuación, se muestran los resultados y análisis de las estimaciones de modelos de elección discreta llevados a cabo para esta investigación.

\section{Características generales}

La muestra total está compuesta por las observaciones de los encuestados de los cuatro sectores. La cantidad de encuestados fue de 176, y 1054 observaciones totales (tabla 2). Se utilizaron tres métodos de recolección de datos: en terreno, a través de correo electrónico, y mediante una carta de invitación a participar dejada en las residencias. 
TABLA 2 Cantidad de encuestados y observaciones

\begin{tabular}{|l|c|c|c|c|}
\hline \multicolumn{1}{|c|}{ SECTOR } & MUJERES & HOMBRES & TOTAL & $\begin{array}{c}\text { NÚMERO DE } \\
\text { OBSERVACIONES }\end{array}$ \\
\hline Vitacura & 32 & 26 & 58 & 348 \\
\hline Santa Rosa & 14 & 6 & 20 & 120 \\
\hline Gran Avenida & 25 & 16 & 41 & 244 \\
\hline Pajaritos & 33 & 22 & 57 & 342 \\
\hline Total & 104 & 70 & 176 & 1.054 \\
\hline
\end{tabular}

FUENTE ELABORACIÓN PROPIA

De los encuestados en Vitacura, cerca del 70\% tiene un ingreso por hogar mayor a $\$ 2.400 .000$. En Santa Rosa, el 80\% de los encuestados tiene un ingreso total menor a $\$ 800.000$. En el caso de Gran Avenida y Pajaritos, el $88 \%$ y $84 \%$, respectivamente, se encuentran entre $\$ 400.000$ y $\$ 2.400 .000$. Dado esto, se consideró que el ingreso bajo es menos de $\$ 400.000$; el ingreso medio entre $\$ 400.000$ y $\$ 2.400 .000$; y el ingreso alto, superior a $\$ 2.400 .000$.

\section{Estimación de modelos}

Se estimaron modelos tipo Logit Multinomial (MNL) con la ayuda del software Biogeme (Bierlaire, 2003). El MNL se construyó incorporando los atributos de las imágenes y algunos parámetros para entender el comportamiento de la variable monetaria.

Propietarios y arrendatarios ven afectada su función de utilidad de distinta manera cuando ocurre un aumento de precio de la vivienda. Por un lado, se espera que dicho aumento tenga un efecto positivo para los propietarios (signo positivo); y por otro, que tenga una relación negativa con los arrendatarios (signo negativo). Para representar de mejor manera esta diferencia con respecto al aumento del precio de la vivienda, la variable precio fue modelada de manera innovadora, incluyendo la aversión al riesgo (loss aversion). De acuerdo con lo explicado por Tversky y Kahneman (1991), cuando una persona ya tiene una situación actual en relación con algún atributo y le reporta mayor desutilidad perder versus la utilidad de ganar, es adverso a la pérdida. En estos casos, la situación actual de una persona con respecto a un atributo se usa como punto de referencia para las estimaciones posteriores.

Se consideraron tres niveles de ingreso: alto, medio y bajo. Además, se agregó una interacción entre el ingreso familiar con los encuestados que declararon ser arrendatarios. En el fondo, cómo cambian las preferencias de los arrendatarios según su nivel de ingreso. Esta relación se hizo solo con los arrendatarios, ya que son los que reflejan la verdadera disposición a pagar. Para los propietarios, el precio refleja un cambio en el valor de su propiedad, por lo que no se interpreta como un valor distinto que pagar por tener un entorno diferente. La función de utilidad para este caso se describe de la siguiente forma:

$$
\begin{aligned}
& V_{j n}=A S C_{j}+\beta_{\text {arboles }} \cdot \text { Arboles } j+\beta_{\text {ciclo }} \cdot \text { Ciclovía }_{j}+\beta_{\text {corr }} \cdot \text { Corredor }_{j}+\beta_{\text {arbusto }} \cdot \text { Arbusto }_{j}
\end{aligned}
$$

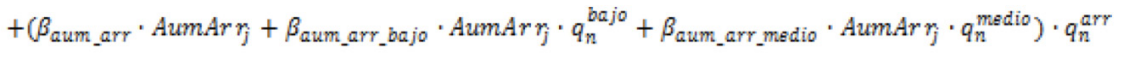

$$
\begin{aligned}
& +\left(\beta_{\text {aum_prop }} \cdot \text { AumProp }_{j}+\beta_{\text {dism_prop }} \cdot \text { DismPro } p_{j}\right) \cdot q_{n}^{\text {prop }}
\end{aligned}
$$


Donde $j$ es un subíndice para la alternativa y $n$ es un subíndice para el individuo encuestado. Los términos $q_{n}^{\text {bajo }}$ y $q_{n}^{\text {medio }}$ son variables dummy que asumen el valor de 1 cuando el individuo $n$ es de ingreso bajo y medio, respectivamente, y cero en caso contrario. Análogamente, las variables dummy $q_{n}^{\text {arr }} \mathrm{y} q_{n}^{\text {prop }}$ indican si el encuestado es arrendatario o propietario respectivamente. Las variables AumArr indican el monto en que aumenta el arriendo en la alternativa $j$ respecto al valor de referencia reportado por el encuestado. De manera similar, las variables Aum prop p $_{\text {y }}$ DismProp in $_{\text {indican }}$ los valores de aumento o disminución en el valor de la propiedad. Se probaron especificaciones incluyendo la variable ${ }^{\text {Dism Arr }}$, que indicaba el monto en que disminuía el arriendo (en caso de hacerlo) para la alternativa $j$; sin embargo, el parámetro para esta variable no resultó ser significativo. Los resultados de las estimaciones se presentan en la tabla 3.

TABLA 3 Resultados estimación modelo tipo Logit Multinomial (MNL)

\begin{tabular}{|l|c|}
\hline \multicolumn{1}{|c|}{ PARÁMETRO } & VALOR $($ TEST-T) \\
\hline$\beta_{\text {aum_arr }}$ & $-0,00721(-2,3)$ \\
\hline$\beta_{\text {aum_arr_bajo }}$ & $-0,0283\left(-1,02^{*}\right)$ \\
\hline$\beta_{\text {aum_arr_medio }}$ & $-0,0183(-2,44)$ \\
\hline$\beta_{\text {aum_prop }}$ & $0,00632(2,69)$ \\
\hline$\beta_{\text {dism_prop }}$ & $-0,00672(-3,32)$ \\
\hline$\beta_{\text {arbol }}$ & $0,843(9,04)$ \\
\hline$\beta_{\text {ciclo }}$ & $0,922(9,6)$ \\
\hline$\beta_{\text {corr }}$ & $0,289(2,71)$ \\
\hline$\beta_{\text {arbusto }}$ & $0,232(2,25)$ \\
\hline & $0,0589\left(0,84^{*}\right)$ \\
\hline log-verosimilitud final & $-593,612$ \\
\hline$N^{\circ}$ de observaciones & 981 \\
\hline
\end{tabular}

$(*)$ NO ES SIGNIFICATIVO AL $95 \%$ DE CONFIANZA.

FUENTE ELABORACIÓN PROPIA

En su mayoría los signos son acordes con lo esperado. Para los atributos árboles, ciclovía y arbustos se esperaba un signo positivo, ya que son atributos que aportan en temas urbanos y que los individuos debieran valorar de manera positiva, de acuerdo con la literatura del urbanismo (Krizec, 2007). El signo del corredor de buses era inesperado, ya que no hay estudios al respecto. Resultó tener un signo positivo, que se podría explicar por la forma y diseño en que se lo presentó en las imágenes. La forma en que se incorporó en la imagen no es el diseño común de los corredores de buses que hay en Santiago. De hecho, se lo incorporó sin segregación y con la pista pintada de rojo, o con arbustos que lo separaban del resto de los 
vehículos. Por esto, podríamos suponer que las personas sí valoran la relación que el corredor establece con su entorno urbano y la posibilidad de incorporar junto a él material vegetal, atributo que en suma contribuye a mejorar la habitabilidad del espacio público de la calle.

Por otro lado, los signos de los dos parámetros asociados al aumento de precio son representativos y están de acuerdo con lo esperado: un signo negativo para los arrendatarios, ya que les reporta desutilidad que suba el precio que tienen que pagar; y un signo positivo en el caso de los propietarios, ya que les reporta utilidad el hecho de que aumente el valor de su propiedad.

$\mathrm{Al}$ incorporar las interacciones entre el precio de arriendo con los ingresos familiares, el modelo se explica mejor, y los resultados ya tienen un sentido concreto. Con estos valores de los parámetros se puede saber la valoración de los atributos urbanos para los tres niveles de ingresos. Se dejó como base el ingreso alto; los parámetros para las personas de ingresos medios y bajos corresponden a la suma entre el parámetro base (ingreso alto) y el parámetro correspondiente a cada ingreso. En la tabla 4 se muestra el parámetro asociado a cada nivel de ingreso.

TABLA 4 | Parámetros asociados a los tres niveles de ingreso

\begin{tabular}{|l|l|}
\hline \multicolumn{1}{|c|}{ NIVEL DE INGRESO } & VALOR \\
\hline$\beta_{\text {ingreso alto }}$ & $-0,00721$ \\
\hline$\beta_{\text {ingreso medio }}$ & $-0,02551$ \\
\hline$\beta_{\text {ingreso bajo }}$ & $-0,03551$ \\
\hline
\end{tabular}

FUENTE ELABORACIÓN PROPIA

Mientras mayor el ingreso familiar, menor es el parámetro. Esto significa que, a pesar de que a todos les provoca desutilidad el aumento del precio, a los de mayores ingresos les afecta o importa menos. Si el análisis se realiza con los parámetros estimados para los propietarios, se encuentra que el parámetro $\beta_{\text {dism_prop, asociado }}$ a una disminución del valor de la propiedad (análogo a un aumento del arriendo en términos de desutilidad), tiene una magnitud similar al de los arrendatarios de ingreso alto. Esto se puede deber a que exista una correlación entre tener un nivel de ingreso alto y ser propietario; sin embargo, la interpretación es similar. El pará-

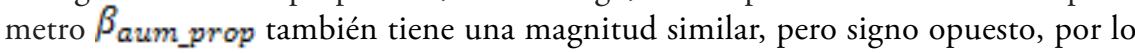
que se puede interpretar que el aumento del valor tiene un efecto equivalente, pero positivo en la utilidad. A pesar de lo anterior, el hecho de que la encuesta haya sido realizada en términos de arriendo y no de valor de las propiedades impide usar estos parámetros para estimar las disposiciones a pagar.

\section{Disposiciones a pagar}

Finalmente, conociendo los valores de los parámetros asociados a cada atributo, se pueden calcular las disposiciones a pagar o willingness-to-pay (WTP). Para el cálculo de la WTP, se consideró el aumento del precio para los arrendatarios. Si 
se considerara la disminución del precio, se interpretaría como una disposición a recibir un monto en dinero por tener o no tener alguno de los atributos urbanos presentes en su entorno. Los resultados presentados en la tabla 5 son considerando los resultados del modelo MNL2, ya que diferencian por nivel de ingreso y son una primera propuesta de WTP proyectos en Chile con características urbanas similares a las de este estudio.

TABLA 5 | Disposiciones a pagar por atributos urbanos

\begin{tabular}{|l|c|c|c|}
\cline { 2 - 4 } \multicolumn{1}{c|}{} & $\begin{array}{c}\text { INGRESO BAJO } \\
\text { (CLP }\end{array}{ }^{[*]} /$ MES $)$ & $\begin{array}{c}\text { INGRESO MEDIO } \\
\text { (CLP/MES })\end{array}$ & $\begin{array}{c}\text { INGRESO ALTO } \\
\text { (CLP/MES })\end{array}$ \\
\hline Árboles & 23.740 & 33.046 & 116.921 \\
\hline Ciclovía & 25.965 & 36.143 & 127.878 \\
\hline Corredor & 8.139 & 11.329 & 40.083 \\
\hline Corredor con arbustos & 14.672 & 20.423 & 72.261 \\
\hline
\end{tabular}

$(*)$ EQUiVAlENCIA MONEDA CHILENA (ClP) A DÓlAR AMERICANO (USD) PARA AGOSTO 20I 5: I USD = 690 CLP. EQUiVAlENCIA MONEDA CHILENA (ClP) A EURo (EUR) PARA AGOSTO 20I 5: I EUR $=773$ CLP.

FUENTE ELABORACIÓN PROPIA

El atributo urbano más valorado es la ciclovía, con una disposición a pagar mensual de $\$ 36.143$ por las personas de ingreso medio; el menos valorado es el corredor de buses, con una disposición a pagar de $\$ 11.329$ por el grupo de ingreso medio.

Es importante mencionar que en los resultados de la encuesta piloto de Vitacura, el corredor tenía un signo negativo, que reflejaba lo que se cree de los corredores actualmente: que no son del gusto de las personas. Sin embargo, estos nuevos resultados, se puede decir que no es el corredor de buses en sí lo que no les gusta, sino que la forma en se relaciona con su contexto urbano inmediato. Además, dada la forma en que se modeló el corredor de buses y arbustos, se pueden sumar sus parámetros y estimar la valoración por el corredor con arbustos segregadores en forma separada del corredor de bus. Así, la disposición a pagar por el corredor con arbustos segregadores pasa a tener un valor casi el doble que por el corredor de buses sin segregación. Esto reafirma la importancia de cómo se diseña el corredor: las personas prefieren un corredor que se integre mejor con su entorno urbano.

En esta misma línea, se debe ser cauto en el uso de estos valores preliminares de WTP para los distintos elementos presentados en este artículo. En primer lugar, estas valoraciones dependen en gran forma de cómo el diseño de la ciclovía o corredor se inserta con su entorno. Por lo tanto, los valores definidos no son extrapolables a cualquier diseńo de ciclovía o corredor, ya que dependen de su diseño y de cómo se inserten en el entorno que los rodea. De hecho, en la ciudad de Santiago no existen actualmente corredores que cuenten con las áreas verdes presentadas en los experimentos. En segundo lugar, este experimento es de preferencias declaradas, no reveladas, lo que podría sobreestimar estas wTP (O’Conor, Johannesson \& Johannesson, 1999).

Como era de esperar, las personas de mayores ingresos tienen mayor disposición al pago por los atributos; de hecho, pagarían hasta cinco veces más que las de 
menores ingresos. Además, diferenciar las disposiciones al pago por los distintos niveles de ingresos, permite a la autoridad establecer un valor por atributo que pueda ser, por ejemplo, la suma ponderada de estos valores.

\section{Discusión y conclusiones}

Esta investigación ofrece un nuevo paradigma, al presentar el transporte como un elemento con un potencial de solucionar problemas de equidad de elementos urbanos. El objetivo de esta investigación era desarrollar una metodología que permitiera identificar y valorar atributos urbanos que mejoran el entorno de proyectos de transporte, para que idealmente fueran incorporados a las próximas evaluaciones sociales de proyectos en Chile. Para esto, se realizó el diseño y aplicación de una encuesta de preferencias declaradas (PD), con la que fue posible derivar las disposiciones al pago por los atributos urbanos. El proceso de creación de la encuesta fue largo e iterativo, ya que desde un comienzo era importante investigar sobre los atributos que se iban a considerar; y al mismo tiempo, había que crear un instrumento adecuado y eficiente para lograr el objetivo de esta investigación.

En particular, se aplicó la metodología en un caso de estudio real en la ciudad de Santiago, considerando áreas verdes, ciclovía y corredor de buses. Las disposiciones a pagar (willingness-to-pay: WTP) se diferenciaron por nivel de ingreso familiar de los encuestados, debido a que los sectores considerados tenían características socioeconómicas distintas entre sí. Para extrapolar estos valores a la ciudad, se aconseja utilizar un único valor (y no separar por sectores de mayor o menor ingreso), para no aumentar la inequidad que ya existe en la ciudad de Santiago respecto a la calidad de infraestructura, preocupación por el entorno y existencia de áreas verdes. Además, los valores obtenidos son preliminares y están sujetos a la presentación de estos elementos en el contexto de la encuesta, dada la relevancia que tiene el cómo se insertan en su entorno.

Para los tres atributos considerados, las wTP fueron positivas y en magnitudes adecuadas a los tres niveles de ingreso. El atributo más valorado resultó ser la ciclovía, considerada en el estudio como la incorporación de una pista en la calzada de color celeste, y separada del flujo vehicular. Una limitación del estudio para el caso de la ciclovía reside en el hecho de que no se sabe cómo cambia esta valoración a medida que el domicilio se aleja del eje donde está la ciclovía. Además, solo se estudió un diseño estándar de ciclovía, y puede que, con distintas características -e.g. ancho, separación y sentido-, sea percibida y valorada de manera diferente por las personas. También puede haber diferencias en la percepción y valoración según la forma en que se inserte la ciclovía con su entorno, pudiendo cambiar estos WTP frente a distintos diseños de ciclovías y de entornos.

En cuanto a los árboles en la vereda, también fueron valorados positivamente por todos los niveles de ingreso. Con esto, se confirma el reconocimiento del arbolado de las calles como un elemento determinante de la habitabilidad del espacio público, tanto en las condiciones directas de confort ambiental (temperatura y asoleamiento) como en la continuidad de los ecosistemas del medioambiente urbano. Pero las áreas verdes no solo fueron consideradas a través del aumento de árboles, sino también 
a través de su acompañamiento a un corredor de buses con arbustos segregadores en su eje. La limitación en la valoración de este atributo recae en la unidad de medición, ya que se consideró como una mejora en verde, sin magnitud, siendo que sería preferible contar con una valoración en metros cuadrados, o cantidad y tipo.

La idea al valorar los corredores de buses es que se incorporen de mejor manera a las características del entorno en el cual se van a construir y puedan aportar sin crear más segregación de la existente. Se valoraron dos tipos de corredores: el primero era un corredor simple sin segregación física con los demás vehículos, que resultó tener una valoración positiva, aunque la menor de entre los atributos considerados. El segundo tipo de corredor incluía arbustos segregadores entre el transporte público y vehículos privados, y resultó tener una valoración mayor que el corredor por sí solo. De hecho, en este último caso la wTP es casi el doble, solo por el hecho de agregarle una banda verde en su diseño. A partir de estos resultados, se puede decir que no es el corredor de buses en sí lo que no les gusta a las personas, sino que la forma en que se inserta con su entorno y la manera en que rompe con las características urbanas y de conexión del sector. Las limitaciones asociadas al diseńo presentado de corredor recaen en características no mostradas; e.g. no se detalla la forma o lugar de los paraderos ni su interacción con el entorno, o tampoco el diseńo de las intersecciones.

Es importante retomar los alcances de esta investigación. En primer lugar, los atributos urbanos involucrados en un proyecto de transporte (como un corredor de buses) son mucho más amplios que los tres valorados en este caso. Pero sí es un aporte importante haber obtenido estas valoraciones de manera preliminar y dar luces sobre cómo se debieran cuantificar los beneficios de proyectos que se integren con su entorno. Es posible que otros atributos sean valorados en futuras investigaciones, pero también existe la posibilidad de extender estos beneficios a otros atributos con características relacionadas. Por ejemplo, en otros países se usan los wider economic impacts o se consideran en la evaluación atributos de manera cualitativa (Mackie et al., 2014). Con los resultados de esta investigación, se pudo corroborar que existen beneficios relacionados al entorno urbano, y quizás se podrían incorporar no solo estos tres con valor monetario, sino que, de manera cualitativa, algunos atributos o características del entorno que aporten de la misma manera.

En segundo lugar, el hecho de haber usado imágenes en la investigación en cierta medida sesga los resultados. Las imágenes no siempre son percibidas de la misma manera por todos. Dejan mucho a la interpretación subjetiva de cada individuo, ya que, al ser estáticas, no pueden transmitir información sobre temperatura, ruido, entre otras cosas.

Por otro lado, la metodología consideró la incorporación de la variable monetaria a través del valor del arriendo, lo que no está directamente relacionado con las variaciones del entorno. Pero resultó ser una medida adecuada, ya que al momento de decidir dónde vivir, las personas toman en cuenta no solo las características físicas de la casa, sino que una parte importante es el barrio, su entorno y sus características de conexión con el resto de la ciudad.

Creemos que nuestros resultados aportan a un inicio de metodologías transporteciudad, y esperamos que puedan ser usadas en las decisiones en materia de políticas públicas, siendo incorporadas a las evaluaciones sociales de proyectos. Una posible 
forma de incorporar estos resultados en materia de políticas públicas sería realizar un experimento similar, que integre los potenciales diseńos de diversos elementos urbanos en el entorno donde se piense emplazar cierto proyecto y calcular las WTP por estos elementos, tomando en cuenta la ubicación de los hogares con respecto al proyecto para determinar cómo la WTP va cambiando según sea la cercanía del hogar con el proyecto, y así determinar su perímetro de influencia.

Quedarían, entonces, como futuras línea de investigación, la valoración de más atributos que describan los espacios públicos - por ejemplo, el espacio peatonal con todos sus componentes de uso y relaciones sociales-, la incorporación de distintas variables que describan más detalladamente cada atributo -e.g. ancho de la ciclovía y calidad de los paraderos en un corredor de buses-, y el perímetro de influencia de estos elementos.

\section{Agradecimientos}

La investigación presentada en este artículo fue financiada por el proyecto "Understanding perception of qualitative attributes in urban space and its effects on user behavior: a mathematical modeling framework" (FONDECYT I I I 30637) y el Centro de Desarrollo Urbano Sustentable, CEDEus (CONICYT/FOndAP I 5 I I OO20).

\section{Referencias bibliográficas}

Ascher, F. (2005). Ciudades con velocidad y movilidad múltiples: un desafío para los arquitectos, urbanistas y políticos. ARQ (Santiago), (60), 11-19.

Ben-Akiva, M. E., \& Lerman, S. R. (1985). Discrete choice analysis: theory and application to travel demand. Series in Transportation Studies, N 9. Cambridge, ma: The miт Press.

Bierlaire, M. (2003). BIOGEME: A free package for the estimation of discrete choice models. Proceedings of the $3^{\text {rd }}$ Swiss Transportation Research Conference, Ascona, Switzerland. http://transp-or.epfl.ch/documents/proceedings/Bier03.pdf

Bonet, L. (2014). Valoración de atributos de barrios patrimoniales desde la perspectiva de sus habitantes. Tesis para optar al grado de Magíster en Ciencias de la Ingeniería. Santiago: Pontificia Universidad Católica de Chile. https://repositorio.uc.cl/bitstream/ handle/11534/16553/000640726.pdf?sequence=1

Borthagaray, A. (2009). Ganar la calle. Compartir sin dividir. Buenos Aires: Ediciones Infinito. Carmona, M., Tiesdell, S., Heath, T., \& Oc, T. (2003). Public places - urban spaces. The dimensions of urban design. Oxford, uK; Burlington, MA: Architectural Press. https:// es.scribd.com/doc/213615854/Public-Places-Urban-Spaces

ChoiceMetrics. (2012). Ngene 1.1.1 USER MANUAL, \& REFERENCE GUIDE: The Cutting Edge in Experimental Design. Accedido desde http://www.choice-metrics. com/documentation.html en enero 2017.

Delbosc, A. (2012). The role of well-being in transport policy. Transport Policy, 23, 25-33. http://doi.org/10.1016/j.tranpol.2012.06.005

Dill, J., \& Carr, T. (2003). Bicycle commuting and facilities in major U.s. cities: If you build them, commuters will use them. Transportation Research Record, 1828, 116-123. https://doi.org/10.3141/1828-14 
Domencich, T. \& McFadden, D. (1975). Urban travel demand: A behavioral analysis. Ámsterdam: North-Holland Publishing Co.

Gehl, J., \& Svarre, B. (2013). How to study public life. Washington, D.c.: Island Press.

Gotschi, T. (2011). Costs and benefits of bicycling investments in Portland, Oregon. Journal of Physical Activity, \& Health, 8 Suppl 1 (Suppl 1), S49-S58. http://www. healthyweld2020.com/assets/7311d20DD1b9CCdc74c8.pdf

Grisolía, J. M., López, F., \& Ortúzar, J. D. D. (2014). Burying the highway: The Social valuation of community severance and amenity. International Journal of Sustainable Transportation, 9(4), 298-309. http://doi.org/10.1080/15568318.2013.769038

Herce, M. (2009). Sobre la movilidad en la ciudad. Propuestas para recuperar un derecho ciudadano. Barcelona: Editorial Reverté.

Herce, M., \& Magrinyà, F. (2013). El espacio de la movilidad urbana. Buenos Aires: Café de las Ciudades.

Hurtubia, R., Guevara, C. A., \& Donoso, P. (2015). Using images to measure qualitative attributes of public spaces through sp surveys. Transportation Research Procedia, 11, 460-474. https://doi.org/10.1016/j.trpro.2015.12.038

Krekel, C., Kolbe, J., \& Wüstemann, H. (2016). The greener, the happier? The effect of urban land use on residential well-being. Ecological Economics, 121, 117-127. http://doi. org/10.1016/j.ecolecon.2015.11.005

Krizec, K. (2007). Estimating the economic benefits of bicycling and bicycle facilities: an interpretive review and proposed methods. En O. Coto-Millán \& V. Inglada (eds.), Essays on Transport Economics (pp. 219-248). Heidelberg / New York: Physica-Verlag Springer Science+Business Media.

Jacobs, J. (1961). The death and life of great american cities. Nueva York: Random House.

Louviere, J. J., Hensher, D. A., \& Swait, J. D. (2000). Stated choice methods: analysis and applications. Cambridge, uk: Cambridge University Press. https://doi.org/10.1017/ CBO9780511753831.008

Mackie, P. J., Jara-Diaz, S., \& Fowkes, A. S. (2001). The value of travel time savings in evaluation. Transportation Research Part E: Logistics and Transportation Review, 37(23), 91-106. https://doi.org/10.1016/S1366-5545(00)00013-2

Mackie, P., Worsley, T., \& Eliasson, J. (2014). Transport appraisal revisited. Research in Transportation Economics, 47, 3-18. http://doi.org/10.1016/j.retrec.2014.09.013

Ministerio de Desarrollo Social, Chile (2013). Resultados Encuesta Casen 2013 [en línea]. http:// www.ministeriodesarrollosocial.gob.cl/resultados-encuesta-casen-2013/

Miralles-Guasch, C. (2002). Ciudad y transporte: el binomio imperfecto. Barcelona: Ariel.

Mullaney, J., Lucke, T., \& Trueman, S. J. (2015). A review of benefits and challenges in growing street trees in paved urban environments. Landscape and Urban Planning, 134, 157-166. http://doi.org/10.1016/j.landurbplan.2014.10.013

Navarro, I. (2016). Valoración de atributos urbanos en un corredor de buses a través de preferencias declaradas. Tesis de Magíster en Ciencias de la Ingeniería, Pontificia Universidad Católica de Chile. https://repositorio.uc.cl/handle/11534/15721

Nelson, A. C., \& Allen, D. (1997). If you build them, commuters will use them. Association between bicycle facilities and bicycle commuting. Transportation Research Record, 1578, 79-83. https://doi.org/10.3141/1578-10 
O‘Conor, R. M., Johannesson, M., \& Johansson, P. O. (1999). Stated preferences, real behaviour and anchoring: some empirical evidence. Environmental and Resource Economics, 13(2), 235-248. https://doi.org/10.1023/A:1008271219089

Ortúzar, J. D. D., Martínez, F. J., \& Varela, F. J. (2000). Stated preferences in modelling accessibility. International Planning Studies, 5(1), 65-85. http://dx.doi. org/10.1080/135634700111828

Ortúzar, J. D. D., \& Willumsen, L. G. (2011). Modelling Transport. Chichester, uk: John Wiley \& Sons. http://doi.org/10.1002/9781119993308

Rueda, S. (2013). El urbanismo ecológico. Urban-e, 004-2013. http://urban-e.aq.upm.es/ articulos/ver/el-urbanismo-ecol-gico/completo

Secretaría de Planificación de Transporte (sectra), Chile. (2014). Actualización y recolección de información del sistema de transporte urbano, IX etapa: Encuesta Origen Destino Santiago 2012. Realizada por Universidad Alberto Hurtado | Observatorio Social. Santiago: SECTRA / Observatorio Social Universidad Alberto Hurtado. http://www.sectra.gob.cl/ biblioteca/detalle1.asp? $\mathrm{mfn}=3253$

Servicios de Impuestos Internos, Chile (2010). Estadisticas y estudios del SII. http://www.sii.cl/ estadisticas/

Sillano, M., \& Ortúzar, J. D. D. (2005). Willingness-to-pay estimation with mixed logit models: Some new evidence. Environment and Planning A, 37(3), 525-550. http:// doi.org/10.1068/a36137

Strazzera, E., Cherchi, E., \& Ferrini, S. (2010). Assessment of regeneration projects in urban areas of environmental interest: A stated choice approach to estimate use and quasi-option values. Environment and Planning A, 42(2), 452-468. http://doi.org/10.1068/a4213

Torres, I., Greene, M., \& Ortúzar, J. D. D. (2013). Valuation of housing and neighbourhood attributes for city centre location: A case study in Santiago. Habitat International, 39, 62-74. http://doi.org/10.1016/j.habitatint.2012.10.007

Tversky, A., \& Kahneman, D. (1991). Loss aversion in riskless choice: a reference-dependent model. The Quarterly Journal of Economics, 106(4), 1039-1061. https://doi. org/10.2307/2937956

Vella-Brodrick, D. a., \& Stanley, J. (2013). The significance of transport mobility in predicting well-being. Transport Policy, 29, 236-242. http://doi.org/10.1016/j. tranpol.2013.06.005 